\title{
Direct experimental determination of spiral spin structures via the dichroism extinction effect in resonant elastic soft $x$-ray scattering
}

\author{
S. L. Zhang, ${ }^{1}$ G. van der Laan, ${ }^{2}$ and T. Hesjedal ${ }^{1, *}$ \\ ${ }^{1}$ Clarendon Laboratory, Department of Physics, University of Oxford, Parks Road, Oxford, OX1 3PU, United Kingdom \\ ${ }^{2}$ Magnetic Spectroscopy Group, Diamond Light Source, Didcot, OX11 ODE, United Kingdom
}

(Received 14 March 2017; published 1 September 2017)

\begin{abstract}
Long-wavelength spin spiral structures are ubiquitous in a large variety of magnetic materials. The detailed magnetic structure can take many variations owing to their different physical origins. Therefore, the unambiguous structural determination is crucial for understanding these spin systems, though such a task is experimentally challenging. Here, we show that ordered spin spiral structures can be fully determined in a single measurement by dichroic resonant elastic $\mathrm{x}$-ray scattering using circularly polarized light. It is found that at certain geometrical conditions, the circular dichroism of the diffraction vanishes completely, revealing a one-to-one correspondence with the spin structure. We demonstrate both theoretically and experimentally this experimental principle, which allows for unambiguous structure determination immediately from the measured signal, whereby no modeling-based data refinement is needed. This largely expands the capabilities of conventional magnetic characterization techniques.
\end{abstract}

DOI: 10.1103/PhysRevB.96.094401

\section{INTRODUCTION}

Spin spiral order is a general type of long-range magnetic order, which can be found in many materials systems. It appears as long-wavelength periodic spin chains in real space, within which the individual spins rotate or precess in a common rotation plane (CRP), forming an incommensurate, modulated magnetic lattice. For most cases, the entire structure breaks inversion symmetry, thus right- and left-handed structures with chirality $\mathcal{C}=1$ and -1 can be found, respectively. The chirality is defined as $\mathcal{C}=\operatorname{sgn}\left[\mathbf{q}_{h} \cdot\left(\mathbf{S}_{i} \times \mathbf{S}_{j}\right)\right]$, where $\mathbf{S}_{i, j}$ are two neighboring spins along the propagation direction, and $\mathbf{q}_{h}$ is the propagation wave vector of the spin modulation.

Spin spiral order can take many different appearances depending on the underlying mechanism. The prototypical systems are rare-earth metals, such as Ho [1], Tb, and Dy [2], in which the long-range Ruderman-Kittel-Kasuya-Yosida interaction is the key ingredient that stabilizes the modulated spin state. The CRP is perpendicular to the propagation wave vector $\mathbf{q}_{h}$, forming a proper-screw helix [3-5]. In noncentrosymmetric chiral magnets such as $\mathrm{MnSi}$ [6], $\mathrm{Fe}_{1-x} \mathrm{Co}_{x} \mathrm{Si}$ [7], $\mathrm{FeGe}$ [8], and $\mathrm{Cu}_{2} \mathrm{OSeO}_{3}$ [9] which are in the cubic space group $P 2{ }_{1} 3$, and $\mathrm{Cr}_{1 / 3} \mathrm{NbS}_{2}$ [10] in the hexagonal space group $P 6_{3} 22$, the bulk-type Dzyaloshinskii-Moriya interaction (DMI) is via a weak spin-orbit coupling. Therefore, due to the competition between the ferromagnetic-type exchange interaction and DMI, long-wavelength helical modulations are formed as the ground state, similar to the magnetic structures found in rare-earth metals. Applying a magnetic field transforms the proper-screw helix into a longitudinal conical spiral in which the spins precess along $\mathbf{q}_{h}$ within the same CRP, however, with

\footnotetext{
*thorsten.hesjedal@physics.ox.ac.uk

Published by the American Physical Society under the terms of the Creative Commons Attribution 4.0 International license. Further distribution of this work must maintain attribution to the author(s) and the published article's title, journal citation, and DOI.
}

a component that is along the propagation direction [6,11]. On the other hand, in ferroelectric materials, the competing superexchange interactions lead to cycloidal-type spin spirals, in which the CRP is parallel to $\mathbf{q}_{h}$, as evidenced in the perovskite magnetites $R \mathrm{MnO}_{3}(R=\mathrm{Tb}, \mathrm{Dy}, \ldots)$ [12,13]. A similar type can be found in thin-film systems as well. For example, in monolayer Fe on $\operatorname{Ir}(111)$ films, the natural inversion symmetry is broken at the surface, resulting in surface DMI. This leads to a cycloidal spin spiral state, exhibiting a long-range-ordered Néel-type domain-wall structure [14]. A similar scenario is also found in multilayered thin-film systems with strong spin-orbit coupling, in which the interfacial DMI plays a role $[15,16]$. Moreover, spinel-type ferroelectric materials such as $\mathrm{CoCr}_{2} \mathrm{O}_{4}$ [17] and Y-type hexaferrite $\mathrm{Ba}_{2} \mathrm{Mg}_{2} \mathrm{Fe}_{12} \mathrm{O}_{22}$ [18] show transverse-conical spin structures, in which the CRP is the same as for the cycloidal type, however, with a net magnetization component that is perpendicular to $\mathbf{q}_{h}$. In another spinel structure, $\mathrm{ZnCr}_{2} \mathrm{Se}_{4}$ [19], the CRP of the helix can be driven by external magnetic field to an arbitrary angle. Furthermore, in Fe pnictides itinerant compounds, the quasinesting of the Fermi surfaces lead to a spin-density-wave state, appearing as the modulation of the itinerant electron spin polarization in a helical fashion [20]. However, the angle of the CRP is more complicated [21]. Similar spin spiral states are also found in bilayer magnetite [22], as well as bubble-domain systems [23], in which spiral order is due to competing interactions within the classic micromagnetic framework [24].

Due to the emerging interest in topologically ordered magnetic materials [25], multiferroic materials [4], as well as Fe-based superconductivity [26], spin spiral structures are drawing enormous attention. The accurate structural determination of the spiral motif is the most crucial experimental task for understanding these systems. In fact, the exact spiral motif structure can be represented by the CRP angle and the conical angle. As shown in Fig. 1(a), we define a base helix with its CRP normal to $\mathbf{q}_{h}$. It takes a right-handed chirality. Based on the base-helix structure, the CRP will have to be rotated about the pitch $(\eta)$ and yaw $(\phi)$ axes [cf. Fig. 1(b)]. Therefore, the angles $(\phi, \eta)$ fully describe the CRP. Next, as shown in 


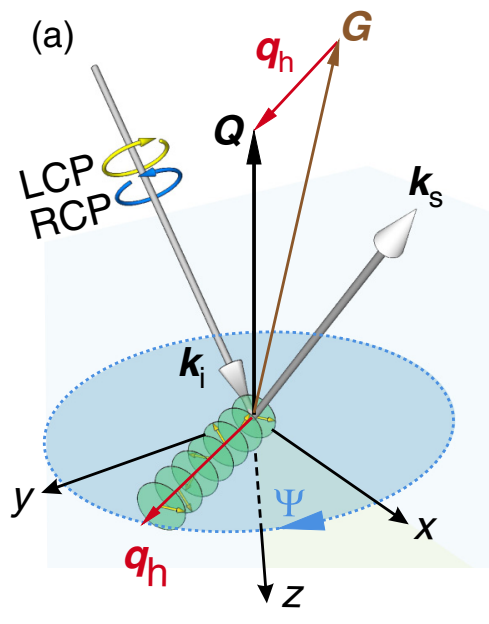

(b)

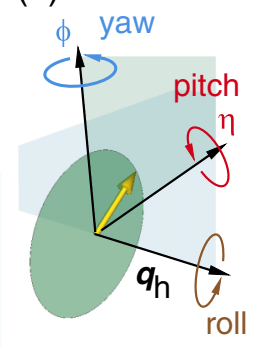

(c)

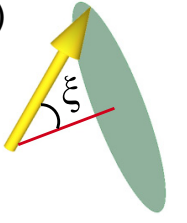

FIG. 1. Measurement geometry. (a) A modulated spin spiral can be represented by a propagation vector $\mathbf{q}_{h}$, and a common rotation plane, in which the spins rotate (green). For the so-called base helix, defined as a right-handed proper-screw-type helix, the surface normal of the common rotation plane is along $\mathbf{q}_{h}$. (b) Depending on the pitch $(\eta)$, yaw $(\phi)$, and cone $(\xi)$ angles that position the common rotation plane in space, all possible types of spin spirals can be described. For measuring the exact structure of the spin spiral, resonant elastic $\mathrm{x}$-ray scattering is used. First, the diffraction condition for $\mathbf{q}_{h}$ has to be fulfilled. Second, the circular dichroism signal is obtained as the intensity difference between left- and right-circularly polarized light illumination. The so-called azimuthal DREXS is performed by taking azimuthal $\Psi$ scans of $\mathbf{q}_{h}$. (c) The conical angle $\xi$ describes the angle between the spin vector and the surface normal of the common rotation plane.

Fig. 1(c), a conical angle $\xi$ is assigned $\left(0^{\circ} \leqslant \xi \leqslant 90^{\circ}\right)$, which spans between spin vector and CRP normal. Thus, using the set of angles $(\phi, \eta, \xi)$, all types of harmonic spin spirals are uniquely represented. In other words, if the three angles can be measured experimentally, the exact spin motif is determined.

Nevertheless, the unambiguous determination of the three angles has remained elusive so far, largely due to the inherent limitations in terms of spatial resolution and/or vectorial magnetization sensitivity of the state-of-the-art magnetic characterization techniques [16,27-30]. On the other hand, neutron [31] and conventional magnetic x-ray diffraction experiments (either in non-resonant or resonant cases) [32-35] accurately measure the modulation wave vector, however, the determination of the motif structure is critically dependent on modelingbased structure factor refinement, assisted by theoretical calculations, often not leading to a unique solution. Here, we show that such spiral spin structures give rise to exclusive dichroic resonant elastic $x$-ray scattering (DREXS) signatures using circularly polarized soft $\mathrm{x}$ rays, from which the CRP angle can be accurately measured. If available, the conical angle $\xi$ can be subsequently retrieved for certain materials.

For magnetic systems, x-ray magnetic circular dichroism has become the foremost technique to study spin and orbital properties of materials, obtainable by applying the magneto-optical sum rules [36]. Distinct from x-ray absorption spectroscopy measurement of standard x-ray magnetic circular dichroism, DREXS may reveal the underlying structural

information for magnetically ordered chiral systems. In the past, several studies have indicated that chiral spin order is closely related to the x-ray magnetic diffraction signals using circularly polarized light [35,37-41]. For example, in patterned ferromagnetic stripes, DREXS measured by comparing the difference in diffracted intensity between left- and rightcircularly polarized incident $\mathrm{x}$ rays, reveals the chirality of the spin domain structure [38]. Also, it was demonstrated using a full polarization analysis of the scattered beam that for multiferroic $\mathrm{TbMnO}_{3}$ the circularly polarized $\mathrm{x}$ rays are sensitive to the handedness of the cycloidal-type spin spiral order [40]. However, chirality is the only structural parameter that can be directly retrieved so far using circularly polarized light for magnetic $\mathrm{x}$-ray diffraction, while the rest of the spiral structural information cannot be determined straightforwardly. Here, we show a generally applied DREXS strategy that not only determines the chirality of the spin spirals, but also fully retrieves the information about the CRP. We demonstrate both theoretically and experimentally that for an arbitrary spin helix, the DREXS surprisingly vanishes at certain geometrical conditions, which are specific signatures of a particular spiral structure. We summarize this phenomenon as the dichroism extinction effect, which can be applied to unambiguously determine the detailed structure of chiral magnetic order, inaccessible to any other methods.

\section{DICHROIC RESONANT ELASTIC X-RAY SCATTERING (DREXS)}

The geometry for performing our DREXS experiment is shown in Fig. 1, in which the resonant elastic X-ray diffraction condition for a scattering wave vector $\mathbf{Q}$ is met. The incommensurate magnetic structure with modulation wave vector $\mathbf{q}_{h}$ appears as satellites around a crystalline lattice peak $\mathbf{G}$ in reciprocal space. $\mathbf{G}$ needs to be oriented along the $z$ axis, while $\mathbf{q}_{h}$ resides within the $x-y$ plane and can be oriented at an arbitrary azimuthal angle $\Psi$. This can be achieved either by rotating the sample azimuthally, or by using a magnetic field that drives the orientation of $\mathbf{q}_{h}$ (assuming the material is susceptible to this field). Consequently, $\mathbf{Q}=\mathbf{G}+\mathbf{q}_{h}$. Here, the dichroic signal, central to the measurement rule, is defined as the diffraction intensity difference in the same geometry using left- and right-circularly polarized incident light (see Appendix). By definition, absorption of a left- (right-) circularly polarized photon changes the magnetic quantum number $m$ of the atom as $\Delta m=-1(\Delta m=+1)[36,42]$. By measuring the DREXS for $\Psi$ varying from $0^{\circ}$ to $360^{\circ}$, an azimuthal circular dichroism profile is obtained. This profile forms the core of our measurement strategy for retrieving the detailed spin motif structure. As derived in Appendix B, the full set of angles $(\phi, \eta, \xi)$ (see Figs. 1 and 2 for an illustration) is explicitly expressed by the azimuthal DREXS intensity

$$
I_{\mathrm{D}}(\Psi)=-Y \sin ^{2} \xi[\cos \theta \sin \eta+\sin \theta \cos \eta \cos (\Psi+\phi)],
$$

where $\theta$ is the incident angle satisfying the diffraction condition for the scattering wave vector $\mathbf{Q}$ that is a known value for an experiment, and $Y$ is a constant. The DREXS intensity follows a sinusoidal curve [see Figs. 2(b), 2(d), 2(f), 2(h), and $2(\mathrm{j})$ ], which goes under certain conditions completely 


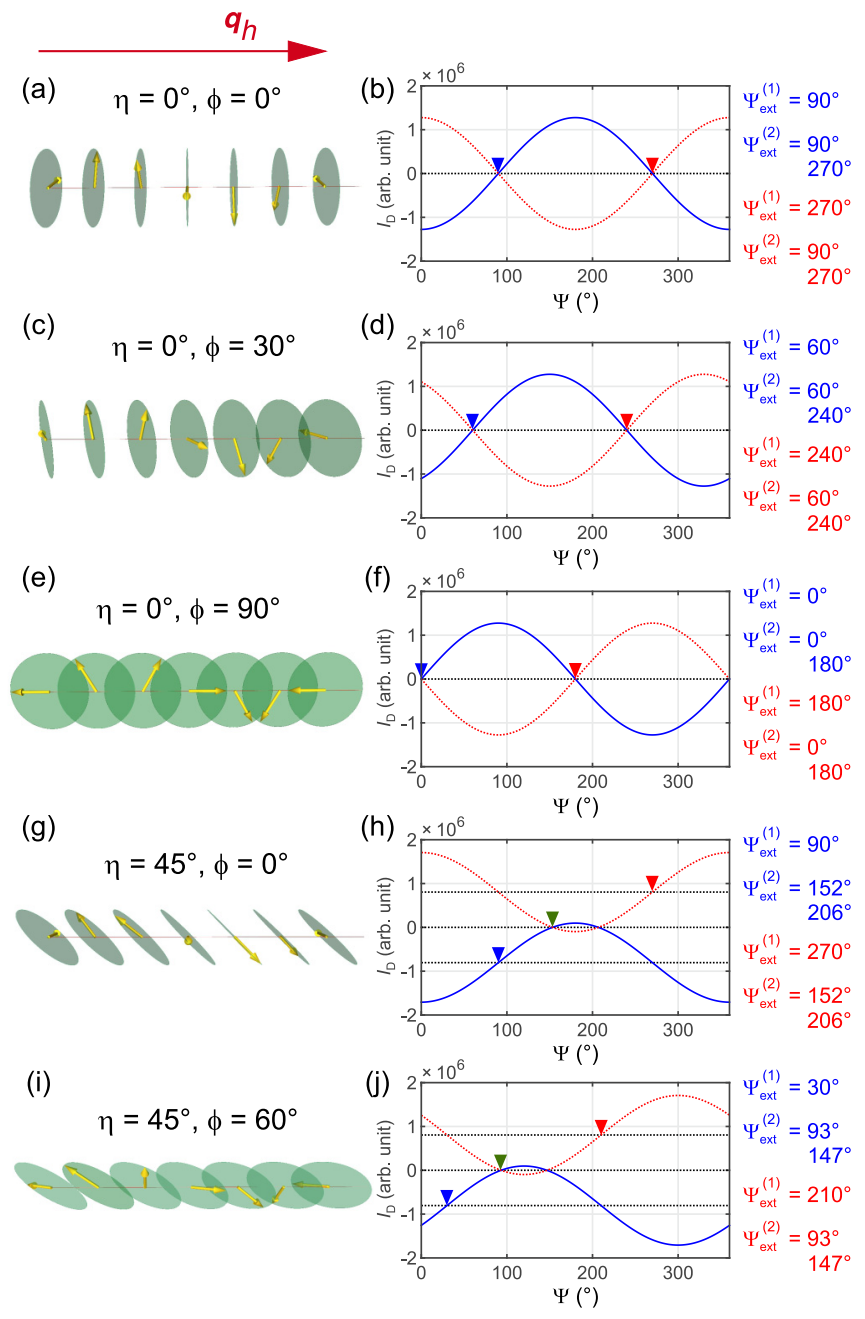

FIG. 2. Correspondence between the common rotation plane and dichroism extinction condition. (a) Spin motif structure with $\eta=0^{\circ}$ and $\phi=0^{\circ}$ (base helix), and (b) corresponding azimuthal DREXS profile (blue curve). The first extinction condition is marked by the blue triangle. The dotted red curve shows the azimuthal DREXS for the opposite chirality, for which the first extinction condition is marked by the red triangle. (c)-(j) Real-space spin motif structures with different $\eta$ and $\phi$ (left-hand side) and corresponding azimuthal DREXS profiles (blue curves, right-hand side). The red curves are the calculated results for real-space spin structures with opposite chirality. For $\eta \neq 0^{\circ}$, the second extinction conditions are marked by the green triangles. On the right-hand side, the numerical values for $\Psi_{\mathrm{ext}}^{(1)}$ and $\Psi_{\mathrm{ext}}^{(2)}$ for both chiralities are listed.

extinct. In the following, we generalize a principle based on Eq. (1), which allows us for determining $(\phi, \eta)$ immediately from the direct measurement result, without any data refinement efforts.

We define the nodes of the sinusoidal curve as the first extinction condition, denoted by

$$
I_{\mathrm{D}}\left(\Psi_{\mathrm{ext}}^{(1)}\right)=I_{\mathrm{D}}^{\text {average }}=I_{\mathrm{D}}^{\max }-I_{\mathrm{D}}^{\min } .
$$

For a more rigorous definition of the extinction conditions, see Appendix C. The yaw angle of the spin motif is given by

$$
\phi=\frac{\pi}{2}-\Psi_{\mathrm{ext}}^{(1)} .
$$

Next, we define the second extinction condition as the azimuthal angle $\Psi_{\mathrm{ext}}^{(2)}$ for which $I_{\mathrm{D}}\left(\Psi_{\mathrm{ext}}^{(2)}\right)=0$. Consequently, the pitch angle $\eta$ is obtained as

$$
\eta=\tan ^{-1}\left[\tan \theta \sin \left(\Psi_{\mathrm{ext}}^{(2)}-\Psi_{\mathrm{ext}}^{(1)}\right)\right] .
$$

Note that there are usually two separate second extinction conditions, both leading to the same $\eta$.

Figure 2(a) shows the base helix motif with $\eta=0^{\circ}$ and $\phi=0^{\circ}$, which forms a three-dimensional periodic spin lattice. The result of the calculation of the DREXS profile is shown in Fig. 2(b) (blue curve). In this case, the sinusoidal curve is symmetric about the $\Psi$ axis. Therefore, $\Psi_{\mathrm{ext}}^{(1)}=\Psi_{\mathrm{ext}}^{(2)}=90^{\circ}$, and $\eta=\phi=0^{\circ}$ is obtained, in agreement with the real-space structure. If the chirality of the spin spiral is reversed, the subsequent dichroic profile will reverse its sign as well. The red curve in Fig. 2(b) is the DREXS curve calculated for the spin structure with opposite chirality. Here, $\Psi_{\mathrm{ext}}^{(1)}=\Psi_{\mathrm{ext}}^{(2)}=270^{\circ}$, leading to $\eta=0^{\circ}, \phi=-180^{\circ}$. Further examples of $\eta=0^{\circ}$ cases are shown in Figs. 2(c)-2(f).

Figure $2(\mathrm{~g})$ shows a spin motif with nonzero $\eta$ canting of the common rotation plane. As a result, the dichroic profile is shifted along the $I_{D}$ axis [blue curve in Fig. 2(h)]. From the DREXS curve, we first find that $\Psi_{\text {ext }}^{(1)}=90^{\circ}$ (blue triangle) by determining the azimuthal angle at the minimum $\left(0^{\circ}\right)$ and then adding $90^{\circ}$, resulting in $\phi=0^{\circ}$. The second extinction condition gives $\Psi_{\mathrm{ext}}^{(2)} \approx 152^{\circ}$ (green triangle). For the calculation, $\theta \approx 48.2^{\circ}$ was used, which is the diffraction condition for the chiral magnet $\mathrm{Cu}_{2} \mathrm{OSeO}_{3}$ [11], resulting in $\eta \approx 45^{\circ}$ which is in excellent agreement with the real-space structure parameters. For the red curve, $\Psi_{\mathrm{ext}}^{(1)}=270^{\circ}$ and $\Psi_{\mathrm{ext}}^{(2)}=152^{\circ}$, leading to $\phi=-180^{\circ}$ and $\eta \approx-45^{\circ}$. This case describes a helix with opposite chirality. The same rules can be applied to other types of spiral structures, as shown in Fig. 2.

\section{AZIMUTHAL DREXS}

The azimuthal DREXS measurements can be summarized using compact two-dimensional maps. In Fig. 3(b), the DREXS profiles for all possible yaw angles for $\eta=0^{\circ}$ spin spirals are plotted. As can been seen, each particular $\phi$ corresponds to a unique DREXS profile. This is also true for all possible $\phi=0^{\circ}$ helices with varying $\eta$, as shown in Fig. 3(c). This one-to-one correspondence represents mathematically a unique relationship between the spin structure and the resulting dichroism extinction conditions.

Next, a more general geometry is discussed. Aside from simply rotating the spiral wave vector $\mathbf{q}_{h}$ azimuthally within the $x-y$ plane, $\mathbf{q}_{h}$ can in fact be oriented in any direction, effectively describing a sphere that is centered at the Bragg peak G. The azimuthal DREXS is the equator of this sphere. Three different types of spin spirals are calculated, and the resulting DREXS spheres are shown in Figs. 3(d), 3(f), and 3(h). For clarity, the corresponding stereographic projections are plotted below in Figs. 3(e), 3(g), and 3(i). First, each of the helices corresponds to a distinct spherical dichroic map. Second, already the equator, i.e., the azimuthal DREXS profile, and thus the associated dichroism extinction conditions, contain the full characteristic DREXS information for an arbitrary spin helix. Therefore, and this is of particular importance from 
(a)
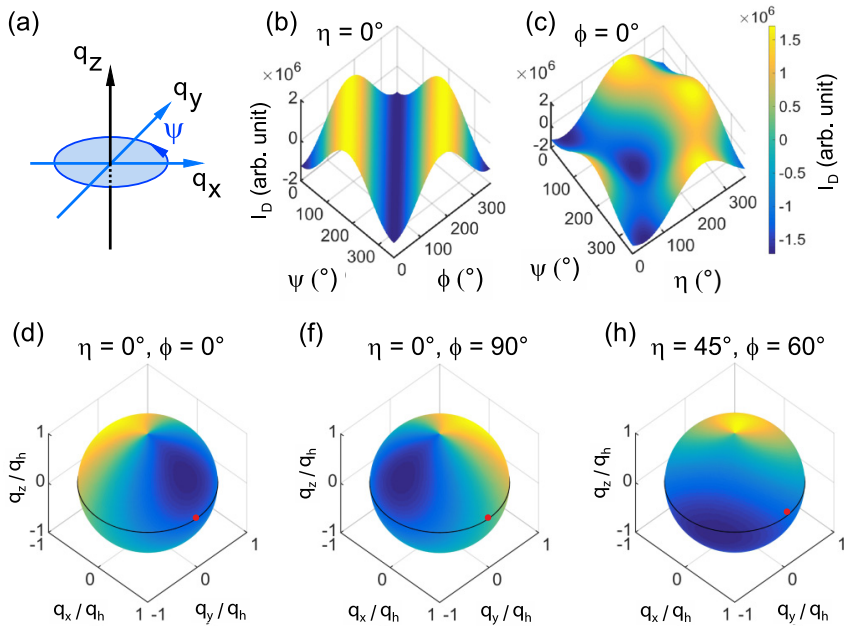

(e)

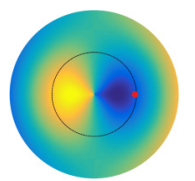

(f) $\eta=0^{\circ}, \phi=90^{\circ}$

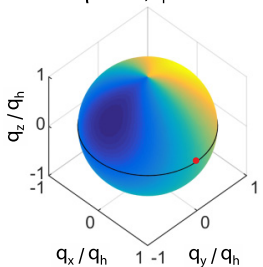

(g)

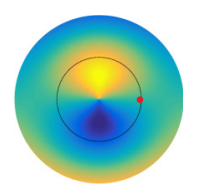

(h) $\eta=45^{\circ}, \phi=60^{\circ}$

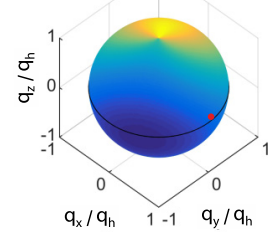

(i)

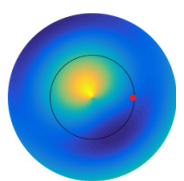

FIG. 3. General features of the dichroism extinction effect. (a) Azimuthal DREXS geometry in which a $\Psi$ scan is performed by rotating $\mathbf{q}_{h}$ over a range of $360^{\circ}$ within the $q_{x}-q_{y}$ plane. (b) For $\eta=0^{\circ}$ spin motif structures, each distinct yaw angle $\phi$ has a unique azimuthal DREXS profile. (c) For $\phi=0^{\circ}$ spin motifs, each pitch angle $\eta$ also has a unique corresponding azimuthal DREXS signal. (d) Circular dichroism sphere for an $\eta=0^{\circ}$ and $\phi=0^{\circ}$ helix structure. Each point $\left(q_{x}, q_{y}, q_{z}\right)$ on the sphere corresponds to an orientation of the spin-helix wave vector. The color encodes the value of the circular dichroism signal. (e) Azimuthal stereographic projection of (d) (top view from above the north pole). The equator, corresponding to the $\Psi$ circle in our experimental geometry, is indicated by the black circle. Red dots mark equivalent points on the sphere and the stereographic projection map. Panels (f), (g) and (h), (i) show equivalent plots for other structures. The azimuthal circular dichroism signal at the equator is unique for each type of helix, thereby greatly simplifying the measurement.

an experimental point of view, performing only azimuthal DREXS scans is sufficient to completely capture the physical properties of this spin system.

\section{ROLE OF THE CONE ANGLE IN DREXS}

Now, we address the role of the cone angle $\xi$ in DREXS measurements. As seen in Eq. (1), $\xi$ only scales the amplitude of the dichroism signal. This means that it is only the CRP that defines a specific type of spin spiral. This is also reflected from the representation of an arbitrary spiral type in Fig. 1. In other words, a conical spiral actually belongs to the same category as the $\xi=90^{\circ}$ helix. Therefore, for an unknown modulated spin structure, first the CRP should be determined, regardless of the value of $\xi$. The influence of $\xi$ is further demonstrated in Fig. 4, in which the azimuthal DREXS profiles for different conical angles are simulated. Indeed, $\xi$ does not affect the fundamental properties specified by the extinction conditions at all, and only the amplitude is scaled. The larger that $\xi$ is, the higher the DREXS signal. At $\xi=0^{\circ}$, a ferromagneticlike order

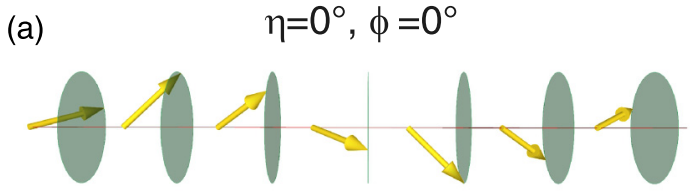

(b)

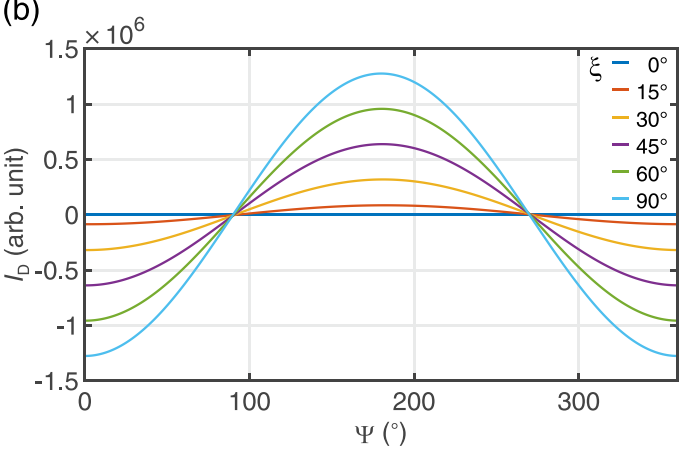

(c) $\eta=45^{\circ}, \phi=60^{\circ}$

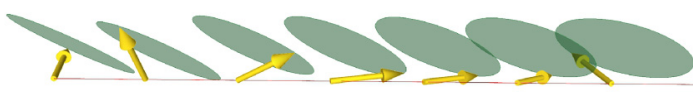

(d)

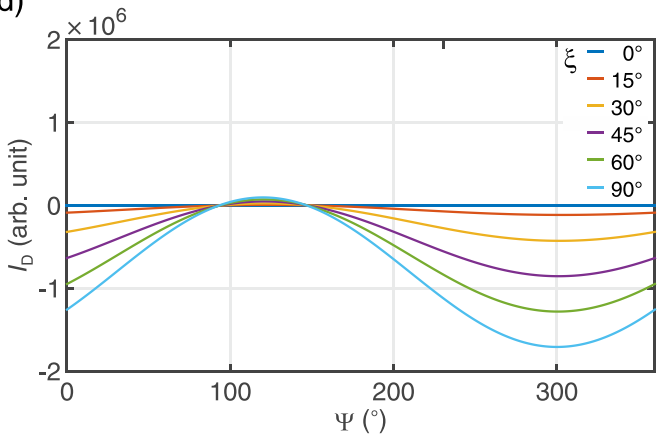

FIG. 4. Conical spirals and their dichroism extinction conditions. (a) A $\eta=0^{\circ}, \phi=0^{\circ}$ conical spiral is essentially of the same type as a proper-screw helix (i.e., the base helix), however, with a $0^{\circ}<\xi<90^{\circ}$ tilt angle. (b) For any allowed $\xi$ angle, the extinction condition for the azimuthal DREXS remains independent of $\xi$. The amplitude of the dichroism indicates the value of $\xi$. These features are universal for all conical structures, as illustrated by another example of a spiral structure in (c) and (d).

is reached, fully smearing out the dichroism signal. Therefore, in order to retrieve the conical angle $\xi$, the comparison of the dichroism signal of a $\xi=90^{\circ}$ helix and a conical spiral state has to be measured. This can usually be achieved by applying an external magnetic field in the $\xi=90^{\circ}$ helical state, if the material is susceptible to the magnetic field. A conical spiral with finite $\xi$ will thus form, with an identical common rotation plane to that of the helical state [25]. Therefore, based on Eq. (1), when performing a measurement at the same azimuthal angle $\Psi$, the measured DREXS signal $I$ for a spin spiral with finite conical angle $\xi$ relates to the $\xi=90^{\circ}$ helical DREXS amplitude $I_{0}$ by $I / I_{0}=\sin ^{2} \xi$. Consequently,

$$
\xi=\sin ^{-1}\left(\sqrt{I / I_{0}}\right) .
$$



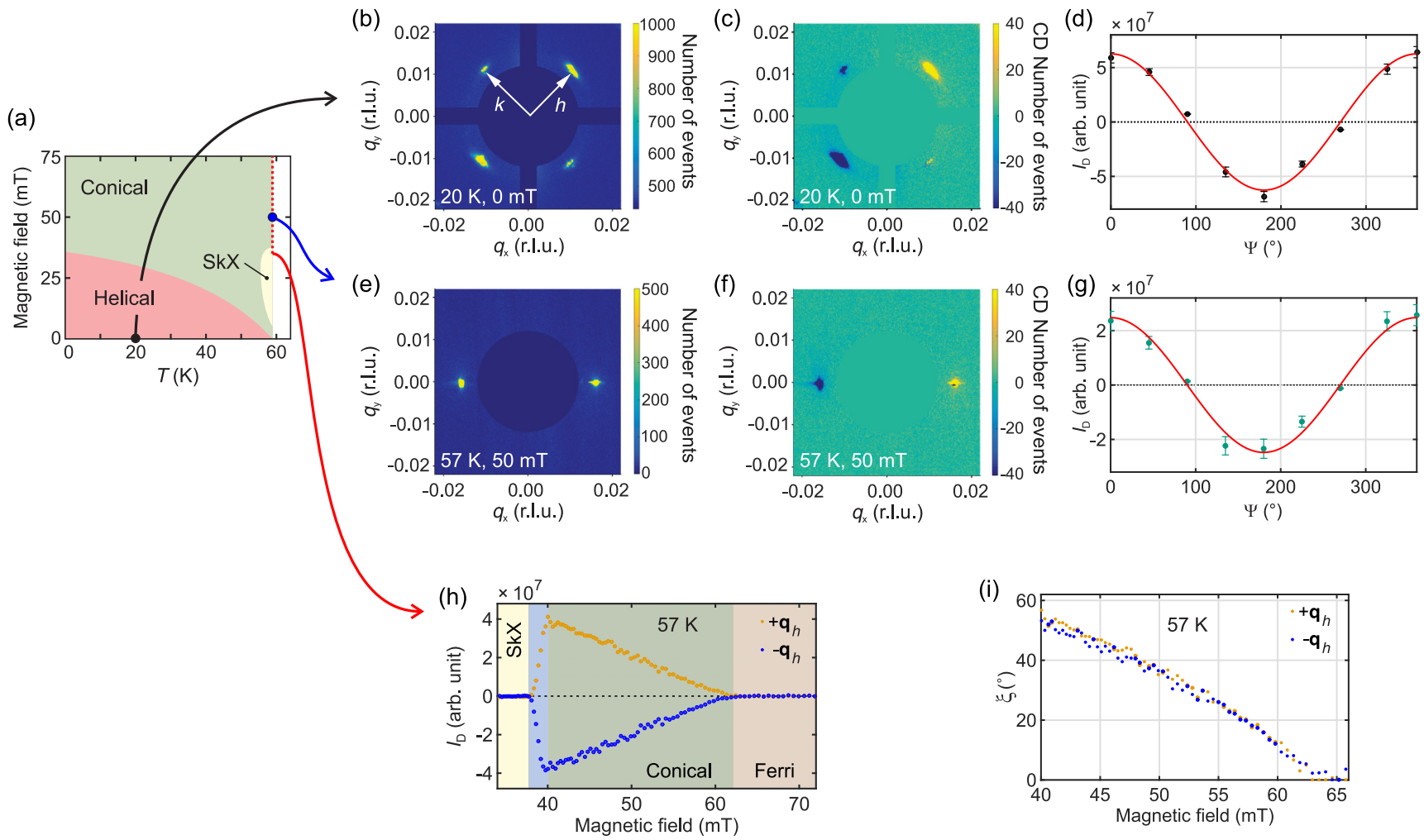

FIG. 5. Experimental demonstration of the dichroism extinction effect. (a) Schematic of the magnetic phase diagram showing the helical, conical, and skyrmion lattice phase $(\mathrm{SkX})$. The $T_{\mathrm{C}}$ is $57.5 \mathrm{~K}$. (b) Reciprocal space map of the helical state of $\mathrm{Cu}_{2} \mathrm{OSeO}_{3}$ at $20 \mathrm{~K}$ and $0 \mathrm{mT}$, measured by resonant x-ray diffraction in the $(h k 1)$ plane. Two pairs of helical peaks are found, resulting from lateral domains. (c) DREXS reciprocal space map in the helical state showing opposite contrast for both partners of a Friedel pair ( $\pm \mathbf{q}_{h}$ ). (d) Azimuthal DREXS plot measured in the helical state. The solid red curve is a fit to the data, using Eq. (2), giving values of $\eta=0^{\circ}$ and $\phi=-180^{\circ}$. (e), (f) Reciprocal space map [in the $(h k 1)$ plane] and circular dichroism map for the conical state, respectively. Note that the magnetic field is applied along the $x$ axis in real space. (g) Azimuthal DREXS plot for the conical state. As in (c), the fit to the data using Eq. (2) yields $\eta=0^{\circ}$ and $\phi=-180^{\circ}$. On the other hand, the reduced amplitude [compared to (c), with identical incident x-ray flux] indicates that this state has a conical spiral structure with finite $\xi$. (h) DREXS as a function of the external field at $57 \mathrm{~K}$, where the $+\mathbf{q}_{h}$ values correspond to $\Psi=0^{\circ}$ and the $-\mathbf{q}_{h}$ values to $\Psi=180^{\circ}$. The magnetic phases are indicated and cover, in order of increasing field, the SkX, conical, and ferrimagnetic (field-polarized) phase. Note that in the phase transition region (blue region), the SkX and the conical state coexist. (i) Evolution of the conical angle $\xi$ as a function of magnetic field. Note that in the helical state, $\xi=90^{\circ}$.

Using the experimental measurement principles specified in Eqs. (3)-(5), the full set of structure parameters $(\phi, \eta, \xi)$ that characterize any given type of spin spiral can be determined.

\section{EXPERIMENTAL VERIFICATION}

Next, we experimentally demonstrate this principle using a $\mathrm{Cu}_{2} \mathrm{OSeO}_{3}$ single crystal. DREXS experiments were carried out in the RASOR diffractometer on beamline I10 (Diamond Light Source, UK). Single crystals of $\mathrm{Cu}_{2} \mathrm{OSeO}_{3}$ were precharacterized by $\mathrm{x}$-ray diffraction and electron backscattering diffraction to confirm the crystalline quality and single chirality. Magnetometry measurements were performed to map out the magnetic phase diagram [see schematic in Fig. 5(a)], showing the helical, conical, and skyrmion phases, consistent with susceptibility and neutron data presented in Refs. $[9,43]$. The polished crystal surface was $(001)$ oriented for the subsequent resonant $\mathrm{x}$-ray scattering measurements. The incident soft $\mathrm{x}$-ray beam with variable polarization was tuned to an energy around the $\mathrm{Cu} L_{3}$ edge. An energy scan across the $\mathrm{Cu}$ edge is shown in Fig. 3(b) in Ref. [44]. The experimental geometry is depicted in Fig. 1(a). The scattered beam is captured by either a CCD camera or a photodiode point detector. The modulated magnetic structure leads to satellites surrounding the structural peaks in reciprocal space. Azimuthal DREXS measurements are performed by varying the incident light polarization, while measuring the diffraction intensities for helical/conical peaks at varying $\Psi$.

$\mathrm{Cu}_{2} \mathrm{OSeO}_{3}$ is a noncentrosymmetric helimagnet that carries long-wavelength spiral spin structures due to the presence of the Dzyaloshinskii-Moriya interaction [9,45]. At zero field, below the magnetic ordering temperature, the system exhibits a multidomain helical state $\left(\xi=90^{\circ}\right)$ with propagation wave vectors $\left[\mathbf{q}_{h} \approx 0.0158\right.$ reciprocal lattice units (r.l.u.)] locked along the $h, k$, and $l$ directions in reciprocal space $[43,46]$.

The REXS data for the helical state were obtained using $\sigma$-polarized incident light. The plot in the ( $h k 1)$ plane, shown in Fig. 5(b), is consistent with other reports in the literature 
$[11,44,47]$. For generating this reciprocal space map, $\omega-2 \theta$ scans were performed $\pm 2.5^{\circ}$ around the (001) peak. The plot in the $(h k 1)$ plane is obtained by processing a series of CCD images, as described in great detail in the Supplemental Material to Ref. [11]. Two sets of Friedel pairs are found within the $(h k 1)$ plane, corresponding to two helical domains, locked by the weak cubic anisotropy.

We then carry out DREXS under the same measurement conditions. For DREXS, RSMs in the $(h k 1)$ plane are obtained for both left- and right-circularly polarized light, and then subtracted to obtain the DREXS reciprocal space map. As shown in Fig. 5(c), a clear difference in dichroism between the partners of each Friedel pair is found. Starting from $\Psi=0^{\circ}$ (the $q_{x}$ axis), the DREXS undergoes a positive $\rightarrow$ negative $\rightarrow$ negative $\rightarrow$ positive transition. From this dichroic pattern, one can immediately determine the chirality of the helix as being left handed [i.e., opposite to the system shown in Fig. 2(a)], by comparison with Figs. 2(b) and 2(d).

Detailed azimuthal DREXS scans were performed by varying the azimuthal angle from $0^{\circ}$ to $360^{\circ}$, sampling on a single helical domain by reducing the beam size. The measured DREXS profile [Fig. 5(d)] is a well-defined sinusoidal curve, normalized to 1 for the helical state, which is almost symmetric about the $\Psi$ axis. This means that $\Psi_{\mathrm{ext}}^{(1)}=\Psi_{\mathrm{ext}}^{(2)}$, leading to $\eta=0^{\circ}$. Further, $\Psi_{\mathrm{ext}}^{(1)} \approx 270^{\circ}$ leading to $\phi \approx-180^{\circ}$. Consequently, by applying our measurement principle, it can be unambiguously concluded that the helical structure is a proper-screw-type helix with left-handed chirality. The fitted result, using the azimuthal DREXS equation with $\phi=-180^{\circ}$ and $\eta=0^{\circ}$ is shown by the red line, which is in excellent agreement with the spin structure model.

By increasing the magnetic field to $50 \mathrm{mT}$ (applied along the $x$ direction), the helical state evolves into a single-domain conical state with a finite $\xi$ angle. The conical phase leads to the well-known twofold-symmetric diffraction pattern in $\sigma$-polarized REXS, as shown in Fig. 5(e). The observed dichroism again suggests that the conical spiral has left-handed chirality, in agreement with the helical state. The measured azimuthal DREXS profile is shown in Fig. $5(\mathrm{~g})$. It bears a large resemblance with that shown in Fig. 5(d), except that in Fig. 5(g) the normalized dichroism amplitude is reduced. By extracting the extinction conditions $\left(\Psi_{\mathrm{ext}}^{(1)}=\Psi_{\mathrm{ext}}^{(2)} \approx 270^{\circ}\right.$ ), we can clearly conclude that the conical state shares the identical common rotation plane to that of the helical state and that the smaller DREXS intensity indicates the presence of a conical $\xi$ angle.

In order to measure the exact conical angle $\xi$, fielddependent DREXS is performed. Figure 5(h) shows the dichroism signal as a function of magnetic field at $\Psi=0^{\circ}$ for the conical Friedel pair $\left(\mathbf{q}_{h}\right.$ and $\left.-\mathbf{q}_{h}\right)$. At $57 \mathrm{~K}$, a finite magnetic field will first stabilize the skyrmion lattice phase, which has no conical peaks (which would be located at different positions in reciprocal space) [11]. With increasing field, the skyrmion lattice phase will undergo a first-order phase transition to the conical state, evidenced by the sudden enhancement of the conical DREXS signal as shown in Fig. 5(h). A further field increase will gradually reduce $\xi$, leading to a reduction of the dichroism signal, according to Eq. (1). A larger field will finally destroy the conical structure, by dragging all the spins to align parallel (field-polarized state), giving rise to zero diffraction intensity at $\pm \mathbf{q}_{h}$ [11]. As a reference, we also measured the helical DREXS signal for a $\xi=90^{\circ}$ system at $\Psi=0^{\circ}$, under identical experimental conditions. This way, based on Eq. (5), the exact value of $\xi$ as a function of magnetic field can be retrieved [cf. Fig. 5(i)]. As a result, the full set of structure parameters $(\phi, \eta, \xi)$ of both the helical and the conical phases of $\mathrm{Cu}_{2} \mathrm{OSeO}_{3}$ were unambiguously determined.

\section{SUMMARY AND CONCLUSIONS}

In summary, we have demonstrated a magneto-optical principle by combining resonant $\mathrm{x}$-ray scattering and circular dichroism. We show that for a particular chiral spin modulation structure, the signal varies with azimuthal rotation, following a well-defined analytical relationship. The systematic extinctions unambiguously reveal the underlying magnetic structure. We formulate experimental rules that can be used to directly determine the spin motif structure with high accuracy, which goes beyond the capabilities of other magnetic characterization techniques.

There are three aspects worth noting for further applying this principle to a wider range of materials. First, our measurement strategy requires that the incommensurate magnetic propagation wave vector lies within the $x-y$ plane, which is probed as magnetic satellite peaks that decorate a structural peak. This is not always the case for soft $\mathrm{X}$-ray resonant scattering, as the wavelength of the incident light is normally long. For materials with shorter lattice constants, such as MnSi and FeGe, no structural peak is accessible in the experimental geometry shown in Fig. 1(c). Nevertheless, one can use the surface diffraction geometry [38,48-50]. Then, the incommensurate spiral peaks appear as magnetic crystalline truncation rods. In this case, at higher incident angles for "longer rods" close to $\theta=45^{\circ}$, the scattering geometry for performing dichroism extinction analysis can be achieved. Therefore, in theory, DREXS is capable of characterizing all types of long-wavelength spin spiral materials regardless of their lattice constants. Second, it is essential to confirm that the magnetic order is of a spin-spiral type before performing the dichroism extinction analysis. In fact, this problem is self-solving by observing a dichroic feature in the magnetic peaks. As illustrated in Figs. 5(c) and 5(f), the Friedel pair of the modulated magnetic peaks will show dichroism, which is the unique feature of spin-spiral-type order. On the other hand, if the magnetic order is not of a spiral type, there is no dichroic difference between the Friedel pairs. In this way, the magnetic structure is determined in one single experiment. Third, it is worth mentioning that the proposed technique for retrieving the conical angle $\xi$ is not applicable to all materials. In fact, only materials hosting $\xi=90^{\circ}$ spirals and where the conical phases are susceptible to an applied magnetic field are suitable for DREXS.

Our experimental strategy can be extended to include nonresonant $\mathrm{x}$-ray magnetic scattering in both the soft and hard x-ray regions with modifications. Although the lightmatter interaction process is fundamentally different for hard $\mathrm{x}$-ray magnetic scattering and nonresonant magnetic $\mathrm{x}$-ray scattering, the geometrical effect is similar [51-53]. Therefore, a similar dichroism extinction effect can be expected. Consequently, this experimental principle is a universal method for the exploration of helical magnetic materials. 


\section{ACKNOWLEDGMENTS}

S.L.Z. was supported by the Semiconductor Research Corporation (SRC) and T.H. acknowledges financial support by EPSRC (Grant No. EP/N032128/1). The resonant soft x-ray scattering experiments were carried out on beamline I10 at the Diamond Light Source, UK, under Proposals No. SI-11784 and No. SI-12958. Technical assistance by M. Sussmuth is gratefully acknowledged. We also thank the I10 team for their ongoing instrumental and measurement support.

\section{APPENDIX A: POLARIZATION DEPENDENCE OF RESONANT ELASTIC X-RAY SCATTERING}

We develop our model starting from the well-established equations for resonant elastic $\mathrm{x}$-ray scattering (REXS) in magnetic systems [39,51-53]. In the electric dipole approximation, for a single magnetic ion at site $n$ that carries a magnetic moment $\mathbf{m}_{n}$, the REXS form factor is given by

$$
f^{\mathrm{res}}=f_{0}\left(\epsilon_{s}^{*} \cdot \epsilon_{i}\right)-i f_{1}\left(\epsilon_{s}^{*} \times \epsilon_{i}\right) \cdot \mathbf{m}_{n},
$$

where $\epsilon_{i}$ and $\epsilon_{s}$ are the incident and scattered light polarization, and $f_{0}$ and $f_{1}$ the energy-dependent charge and linear magnetic term, respectively. At fixed photon energy, the factors $f_{0}$ and $f_{1}$ become constant complex numbers. Higher-order terms can be neglected here as they do not contribute to the measured signals in the proposed method. The scattering intensity $I(\mathbf{q})$ for an incommensurate spin lattice, in the first Born approximation, can then be written as [39]

$$
\begin{gathered}
I(\mathbf{q})=\left|\mathcal{F}_{1}^{\mathrm{res}}(\mathbf{q})\right|^{2}, \quad \text { with } \\
\mathcal{F}_{1}^{\mathrm{res}}(\mathbf{q})=-i \int d \mathbf{r}^{2} f_{1}\left(\epsilon_{s}^{*} \times \epsilon_{i}\right) \cdot \mathbf{m}(\mathbf{r}) e^{i \mathbf{q} \cdot \mathbf{r}} \\
=-i F_{1}\left(\epsilon_{s}^{*} \times \epsilon_{i}\right) \cdot \mathbf{M}(\mathbf{q}),
\end{gathered}
$$

where $\left|F_{1}\right|$ is the Fourier transform of $f_{1}$, and $\mathbf{M}(\mathbf{q})$ the Fourier transform of the real-space magnetization configuration $\mathbf{m}(\mathbf{r})$. Here, the continuum approximation is used such that $\mathbf{m}(\mathbf{r})$ can be regarded as a continuous vector field of the magnetization.

Next, the dependence on $\epsilon_{s}$ and $\epsilon_{i}$ is considered. As will be shown below, using the density matrix approach, the specific scattered light polarization $\epsilon_{s}$ does not play a role since $I(\mathbf{q})$ is summed over all values of $\epsilon_{s}$. The geometry used to carry out the polarization-dependent scattering cross section is illustrated in Fig. 1 in the main text. The choice of Cartesian coordinates is governed by the scattering plane, i.e., the $x-z$ plane is parallel to the scattering plane, and the $x-y$ plane is perpendicular to the diffraction wave vector $\mathbf{Q}$. This also defines the reciprocal space $\left(q_{x}, q_{y}, q_{z}\right)$. Therefore, $\mathbf{k}_{i}=k(\cos \theta, 0, \sin \theta), \mathbf{k}_{s}=k(\cos \theta, 0,-\sin \theta)$, and $\mathbf{k}_{s} \times \mathbf{k}_{i}=$ $k(0,-2 \cos \theta \sin \theta, 0)$, where $k$ is the length of the $\mathrm{x}$-ray wave vector (given by the photon energy) and $\theta$ is the Bragg angle satisfying the diffraction condition for the wave vector $\mathbf{Q}$.

We then define the $2 \times 2$ density matrix $\mu$ for the light polarization as

$$
\mu=\frac{1}{2}\left(P_{0} \sigma_{0}+\mathbf{P} \cdot \sigma\right)=\frac{1}{2}\left(\begin{array}{cc}
P_{0}+P_{1} & P_{2}-i P_{3} \\
P_{2}+i P_{3} & P_{0}-P_{1}
\end{array}\right),
$$

where $\sigma_{0}=\left(\begin{array}{ll}1 & 0 \\ 0 & 1\end{array}\right)$, and $\sigma$ are the Pauli matrices $\sigma_{1}=$ $\left(\begin{array}{cc}1 & 0 \\ 0 & -1\end{array}\right), \sigma_{2}=\left(\begin{array}{ll}0 & 1 \\ 1 & 0\end{array}\right), \sigma_{3}=\left(\begin{array}{cc}0 & -i \\ i & 0\end{array}\right) . \mathbf{P}=\left(P_{1}, P_{2}, P_{3}\right)$ is the Poincaré-Stokes representation of the light polarization. For circularly polarized light, $P_{0}=1, P_{1}=0, P_{2}=0$, $P_{3}= \pm 1$.

The density matrix $\mu=\sum_{\lambda}|\lambda\rangle p_{\lambda}\langle\lambda|$ specifies the polarization state for the incident light, with the degree of polarization $\mathcal{P}=\langle\sigma\rangle=\operatorname{Tr}[\sigma \cdot \mu]$, where $|\lambda\rangle$ is the incident polarization eigenstate, with probability $p_{\lambda}$, and Tr stands for trace. The polarization state for the scattered light can then be written as $\mu_{s}=\mathcal{F} \cdot \mu \cdot \mathcal{F}^{\dagger}$, where $\mathcal{F}$ is the scattering form factor. The scattering cross section is obtained by summing over all scattered light polarizations

$$
I(\mathbf{Q})=\operatorname{Tr}\left[\mu_{s}\right]=\operatorname{Tr}\left[\mathcal{F} \cdot \mu \cdot \mathcal{F}^{\dagger}\right] .
$$

Evaluation of Eq. (A5), using $\mathcal{F}$ and $\mu$ given in Eqs. (A3) and (A4), yields [39]

$$
\begin{aligned}
I(\mathbf{Q})= & \frac{1}{2}\left|F_{1}\right|^{2}\left(P_{0}+P_{1}\right)\left|\mathbf{k}_{s} \cdot \mathbf{M}(\mathbf{Q})\right|^{2} \\
& +\frac{1}{2}\left|F_{1}\right|^{2}\left(P_{0}-P_{1}\right)\left[\left|\mathbf{k}_{i} \cdot \mathbf{M}(\mathbf{Q})\right|^{2}+\left|\left(\mathbf{k}_{s} \times \mathbf{k}_{i}\right) \cdot \mathbf{M}(\mathbf{Q})\right|^{2}\right] \\
& -\left|F_{1}\right|^{2} \operatorname{Re}\left\{\left(P_{2}+i P_{3}\right)\left[\mathbf{k}_{s} \cdot \mathbf{M}^{*}(\mathbf{Q})\right]\left(\mathbf{k}_{s} \times \mathbf{k}_{i}\right) \cdot \mathbf{M}(\mathbf{Q})\right\},
\end{aligned}
$$

in which $\mathbf{k}_{i}$ and $\mathbf{k}_{s}$ are tuned to fulfill the diffraction condition for $\mathbf{Q}$. Here, we use the diffraction wave vector $\mathbf{Q}$ as the argument, as it relates to the vector sum over the Bragg peak $\mathbf{G}$ and spin spiral propagation wave vector $\mathbf{q}_{h}$ by $\mathbf{Q}=\mathbf{G}+\mathbf{q}_{h}$.

Plugging $\mathbf{P}=(1,0,0, \pm 1)$ into Eq. (A6) gives the circular dichroism in the REXS condition (i.e., DREXS) at $\mathbf{Q}$ as

$$
I_{\mathrm{D}}(\mathbf{Q})=2\left|F_{1}\right|^{2} \operatorname{Im}\left\{\left[\mathbf{k}_{s} \cdot \mathbf{M}^{*}(\mathbf{Q})\right]\left(\mathbf{k}_{s} \times \mathbf{k}_{i}\right) \cdot \mathbf{M}(\mathbf{Q})\right\} .
$$

\section{APPENDIX B: CIRCULAR DICHROISM FOR AN ARBITRARY SPIN MODULATION}

We first define the base helix of the motif as a proper-screw helix with right-handed chirality. $\mathbf{q}_{h}$ propagates along the $x$ direction as shown in Fig. 2(a) in the main text. It is expressed as

$$
\begin{aligned}
& m_{1}=M_{S} \cos \xi, \\
& m_{2}=M_{S} \sin \xi \cos \left(\mathbf{q}_{h} \cdot \mathbf{r}\right), \\
& m_{3}=M_{S} \sin \xi \sin \left(\mathbf{q}_{h} \cdot \mathbf{r}\right),
\end{aligned}
$$

where $\mathbf{m}=\left(m_{1}, m_{2}, m_{3}\right), M_{S}$ is the saturation magnetization, and $\xi$ is the cone angle. Note that there should also be $\cos \left(\mathbf{q}_{h} \cdot \mathbf{r}+\kappa\right)$ and $\sin \left(\mathbf{q}_{h} \cdot \mathbf{r}+\kappa\right)$ terms in Eq. (B1) that describe the real-space phase shift $\kappa$ of the spins within the motif. This will also appear in the expressions of the Fourier transforms. However, this phase information will vanish when calculating the circular dichroism intensity, obtained by inserting the expressions into Eq. (A7). The underlying reason for their disappearance is that the scattering amplitudes are squared (the famous "phase problem"), resulting in the same values for $\kappa$, independent of the phase. Therefore, without loss of generality, $\kappa=0$ can be chosen to simplify the derivation.

Next, the rotations $\eta$ and $\phi$ are applied to the helix. This is equivalent to the combined rotation operation $\mathcal{R}_{\phi} \mathcal{R}_{\eta} \mathbf{m}$, 
where

$$
\mathcal{R}_{\phi}=\left(\begin{array}{ccc}
\cos \phi & -\sin \phi & 0 \\
\sin \phi & \cos \phi & 0 \\
0 & 0 & 1
\end{array}\right)
$$

and

$$
\mathcal{R}_{\eta}=\left(\begin{array}{ccc}
\cos \eta & 0 & -\sin \eta \\
0 & 1 & 0 \\
\sin \eta & 0 & \cos \eta
\end{array}\right) .
$$

Thereafter, the helix can be written as

$$
\begin{aligned}
m_{1}= & -M_{S} \sin \xi \cos \phi \sin \eta \sin \left(\mathbf{q}_{h} \cdot \mathbf{r}\right) \\
& -M_{S} \sin \xi \sin \phi \cos \left(\mathbf{q}_{h} \cdot \mathbf{r}\right)+C_{1}, \\
m_{2}= & -M_{S} \sin \xi \sin \phi \sin \eta \sin \left(\mathbf{q}_{h} \cdot \mathbf{r}\right) \\
& +M_{S} \sin \xi \cos \phi \cos \left(\mathbf{q}_{h} \cdot \mathbf{r}\right)+C_{2}, \\
m_{3}= & M_{S} \sin \xi \cos \eta \sin \left(\mathbf{q}_{h} \cdot \mathbf{r}\right)+C_{3},
\end{aligned}
$$

where $C_{1}, C_{2}$, and $C_{3}$ are a function of $\xi$, and independent of $\mathbf{r}$. When the Fourier transform is performed, these terms become $\delta$ functions, which are only nonzero at the origin of reciprocal space $\mathbf{q}=0$. Thus, their detailed functions are not relevant at this point.

In our azimuthal geometry for the diffractive XMCD, $\mathbf{q}_{h}$ is required to propagate within the $q_{x}-q_{y}$ plane. We define $\Psi=0^{\circ}$ as the angle where $\mathbf{q}_{h}$ is along the positive $q_{x}$ direction. In real space, this corresponds to the helix pitch being oriented along $x$. Therefore, in our scattering coordinate system, a rotation of the helix by $\Psi$ will give rise to the magnetization profile of $\mathcal{R}_{\Psi} \mathbf{m}$ (see Fig. 1 in the main text), where

$$
\mathcal{R}_{\Psi}=\left(\begin{array}{ccc}
\cos \Psi & -\sin \Psi & 0 \\
\sin \Psi & \cos \Psi & 0 \\
0 & 0 & 1
\end{array}\right) .
$$

To meet the diffraction condition $\mathbf{Q}=\mathbf{G}+\mathbf{q}_{h}$ at $\Psi, \mathbf{Q}$ has to be brought into the scattering plane. In a common four-circle diffractometer, this is achieved by compensating the diffraction offset by the other two rotation axes, i.e., the $\mathcal{K}$ axis, which is perpendicular to both the $\Omega$ axis (i.e., the main axis rotates within the scattering plane) and the $\Psi$ axis, as well as the $\Omega$ axis. Consequently, the components of the magnetic structure transform into

$$
\left(\begin{array}{l}
m_{1}^{\prime} \\
m_{2}^{\prime} \\
m_{3}^{\prime}
\end{array}\right)=\mathcal{R}_{\Omega} \mathcal{R}_{\mathcal{K}}\left(\begin{array}{l}
m_{1} \\
m_{2} \\
m_{3}
\end{array}\right),
$$

where $\mathcal{R}_{\mathcal{K}}$ and $\mathcal{R}_{\Omega}$ are the rotation matrices for the $\mathcal{K}$ and $\Omega$ axes. The combination of these two rotations brings $\mathbf{Q}$ into the scattering plane for fulfilling the diffraction condition.

However, it is essential to note that this change would be negligible for most of the long-wavelength modulated magnetic structures. For instance, $q a=0.0158$ for $\mathrm{Cu}_{2} \mathrm{OSeO}_{3}$ (cf. Ref. [11]), where $a$ is the lattice constant. Therefore, for $\mathbf{G}=(0,0,1)$ and $\mathbf{Q}=\mathbf{G}+\mathbf{q}_{h}$, the change of both $\mathcal{K}$ and $\Omega$ is less than $0.9^{\circ}$ for all $\Psi$ angles. This makes the effect of $\mathcal{R}_{\mathcal{K}}$ and $\mathcal{R}_{\Omega}$ negligible as well. In the long-wavelength approximation, we can then take

$$
\left(\begin{array}{l}
m_{1}^{\prime} \\
m_{2}^{\prime} \\
m_{3}^{\prime}
\end{array}\right) \approx\left(\begin{array}{l}
m_{1} \\
m_{2} \\
m_{3}
\end{array}\right)
$$

Consequently, the Fourier transform for $\mathbf{q}=\mathbf{q}_{h}$ yields

$$
\begin{aligned}
M_{1}\left(\mathbf{q}_{h}\right)= & -\pi M_{S} \sin \xi \sin (\Psi+\phi) \\
& +i \pi M_{S} \sin \xi \sin \eta \cos (\Psi+\phi), \\
M_{2}\left(\mathbf{q}_{h}\right)= & \pi M_{S} \sin \xi \cos (\Psi+\phi) \\
& +i \pi M_{S} \sin \xi \sin \eta \sin (\Psi+\phi), \\
M_{3}\left(\mathbf{q}_{h}\right)= & -i \pi M_{S} \sin \xi \cos \eta .
\end{aligned}
$$

Plugging this result into Eq. (A7) yields the expression for azimuthal DREXS, shown as Eq. (1) above, where $Y=4\left|F_{1}\right|^{2} \pi^{2} k^{2} M_{S}^{2} \cos \theta \sin \theta$. This equation describes the functional dependence of the DREXS signal on the azimuthal rotation of the helix, and its proportionality to the combination of certain helix parameters. The azimuthal DREXS profile is essentially a sinusoidal curve with a period of $2 \pi$. The yaw angle $\phi$ will effectively shift the sinusoidal profile along $\Psi$, and the pitch angle $\eta$ will introduce an asymmetry of the sinusoidal curve. Therefore, by fitting the measured azimuthal DREXS profile to Eq. (1), the common rotation plane of the helix motif can be unambiguously determined.

\section{APPENDIX C: DEFINITION OF THE FIRST AND SECOND DICHROISM EXTINCTION CONDITIONS}

First, let us first assume $\eta=0^{\circ}$ and $\phi=0^{\circ}$, i.e., a base helix as shown in Fig. 2(a). The results of the numerical calculation for the azimuthal DREXS profile are plotted as the blue curve in Fig. 2(b). This cosine curve is in agreement with the results obtained from Eq. (1). There are two characteristic points, $\Psi=90^{\circ}$ and $270^{\circ}$, at which the dichroism vanishes. If the chirality of the helix is reversed, the sign of the DREXS profile is inverted [shown as the red curve in Fig. 2(b)].

Let us define the first extinction condition $\Psi_{\mathrm{ext}}^{(1)}$, such that $\partial I_{\mathrm{D}} /\left.\partial \Psi\right|_{\Psi=\Psi_{\text {ext }}^{(1)}}>0$, meaning that it satisfies two conditions: (1) $I_{\mathrm{D}}\left(\Psi_{\text {ext }}^{(1)}\right)=0$ and (2) $I_{\mathrm{D}}$ should always evolve from negative to positive when crossing over $\Psi_{\mathrm{ext}}^{(1)}$. Therefore, in this case, $\Psi_{\mathrm{ext}}^{(1)}=90^{\circ}$ for the blue curve. It is then obvious that the structure parameter $\phi$ for the helix directly relates to the first extinction condition by $\phi=\pi / 2-\Psi_{\mathrm{ext}}^{(1)}=0^{\circ}$, which agrees with the numerical results. On the other hand, for the red curve, $\Psi_{\mathrm{ext}}^{(1)}=270^{\circ}$, giving rise to $\phi=-180^{\circ}$. This suggests that the real-space base helix in Fig. 2(a) has a yaw angle which is rotated by $-180^{\circ}$ azimuthally, essentially equivalent to the reversal of its chirality. Therefore, the first extinction condition gives an unambiguous value for the yaw angle.

Next, we add a $\phi$ rotation, while keeping $\eta=0^{\circ}$. In this case,

$$
\begin{aligned}
I_{\mathrm{D}} & \propto-\cos (\Psi+\phi), \text { with the first extinction condition } \\
\phi & =\frac{\pi}{2}-\Psi_{\mathrm{ext}}^{(1)} .
\end{aligned}
$$

This example is illustrated in Figs. 2(c) and 2(b), in which the base helix has a yaw canting. The numerical calculation [Fig. 2(b)] shows that $\Psi_{\mathrm{ext}}^{(1)}=60^{\circ}$, which further gives $\phi=$ $30^{\circ}$. This is in excellent agreement with the results shown in Fig. 2(c). Another example with $\phi=90^{\circ}$ and $\Psi_{\mathrm{ext}}^{(1)}=0^{\circ}$ is shown in Figs. 2(e) and 2(f). 
Finally, the rotation about $\eta$ is included. First, as shown in Eq. (1), one can ignore the contribution from $\theta$, as this is a constant which only relates to the absolute diffraction condition. Second, the direct consequence of adding the $\eta$ degree of freedom is a scaling and shifting of the cosine curve, while the periodicity and phase of the profile is not changing (equivalent to $\eta=0^{\circ}$ ). In other words, the determination of $\phi$ using the first extinction condition, given by Eq. (C1), is unaffected. In comparison to the previous cases, in order to locate $\Psi_{\text {ext }}^{(1)}$, the sinusoidal curve needs to be shifted vertically to make the curve symmetric about the horizontal axis. Alternatively, the maxima or minima of the DREXS curve can be determined and $\Psi_{\text {ext }}^{(1)}$ is obtained by adding or subtracting $\pi / 2$ to those values.

The second extinction condition $\Psi_{\mathrm{ext}}^{(2)}$ is defined by $I_{\mathrm{D}}=0$. Now, $\eta$ is taken into account right from the start. This results in

$$
\begin{aligned}
\cos \theta \sin \eta & =-\sin \theta \cos \eta \cos \left(\Psi_{\mathrm{ext}}^{(2)}+\phi\right), \text { therefore } \\
\tan \eta & =\tan \theta \sin \left(\Psi_{\mathrm{ext}}^{(2)}-\Psi_{\mathrm{ext}}^{(1)}\right) .
\end{aligned}
$$

Here, $\eta=\tan ^{-1}\left[\tan \theta \sin \left(\Psi_{\mathrm{ext}}^{(2)}-\Psi_{\mathrm{ext}}^{(1)}\right)\right]$ uniquely specifies the pitch angle of the generalized helix. Note that both $\Psi_{\mathrm{ext}}^{(2)}$ extinction points lead to the same $\eta$.

The results for this example are shown in Figs. 2(g)-2(j), in which $\eta=45^{\circ}$ effectively lowers the blue sinusoidal curve. First, the first extinction condition $\Psi_{\mathrm{ext}}^{(1)}=90^{\circ}$ is identified (labeled by the blue triangle). This is obtained by shifting the $I_{\mathrm{D}}$ profile vertically to obtain a symmetric function, giving $\phi=0^{\circ}$. Second, $\Psi_{\mathrm{ext}}^{(2)} \approx 152^{\circ}$ and $206^{\circ}$ are directly obtained from the unshifted curve (marked by the green triangles). In our calculation, we use a value of $\theta \approx 48.2^{\circ}$, which gives the diffraction condition for $\mathrm{Cu}_{2} \mathrm{OSeO}_{3}$. This leads to $\eta \approx 45^{\circ}$ [using Eq. (C2)], in agreement with the real-space structure. Note that the minor difference is due to the numerical errors in the calculation. For the red curve, $\Psi_{\text {ext }}^{(1)}=270^{\circ}$, leading to $\phi=-180^{\circ}$ and $\eta \approx-45^{\circ}$. These parameters describe a helix with opposite chirality of that shown in Fig. 2(c). The same exercise can be carried out for the spirals in Figs. 2(g) and 2(h), which further confirms the validity of this method.
[1] D. Gibbs, D. R. Harshman, E. D. Isaacs, D. B. McWhan, D. Mills, and C. Vettier, Phys. Rev. Lett. 61, 1241 (1988).

[2] J. Jensen and A. K. Mackintosh, Rare Earth Magnetism (Oxford University Press, Oxford, 1991).

[3] T. Kimura, Annu. Rev. Mater. Res. 37, 387 (2007).

[4] Y. Tokura and S. Seki, Adv. Mater. 22, 1554 (2010).

[5] Y. Tokura, S. Seki, and N. Nagaosa, Rep. Prog. Phys. 77, 076501 (2014).

[6] S. Mühlbauer, B. Binz, F. Jonietz, C. Pfleiderer, A. Rosch, A. Neubauer, R. Georgii, and P. Böni, Science 323, 915 (2009).

[7] X. Z. Yu, Y. Onose, N. Kanazawa, J. H. Park, J. H. Han, Y. Matsui, N. Nagaosa, and Y. Tokura, Nature (London) 465, 901 (2010).

[8] X. Z. Yu, N. Kanazawa, Y. Onose, K. Kimoto, W. Z. Zhang, S. Ishiwata, Y. Matsui, and Y. Tokura, Nat. Mater. 10, 106 (2011).

[9] S. Seki, X. Z. Yu, S. Ishiwata, and Y. Tokura, Science 336, 198 (2012).

[10] Y. Togawa, T. Koyama, K. Takayanagi, S. Mori, Y. Kousaka, J. Akimitsu, S. Nishihara, K. Inoue, A. S. Ovchinnikov, and J. Kishine, Phys. Rev. Lett. 108, 107202 (2012).

[11] S. L. Zhang, A. Bauer, D. M. Burn, P. Milde, E. Neuber, L. M. Eng, H. Berger, C. Pfleiderer, G. van der Laan, and T. Hesjedal, Nano Lett. 16, 3285 (2016).

[12] T. Kimura, T. Goto, H. Shintani, K. Ishizaka, T. Arima, and Y. Tokura, Nature (London) 426, 55 (2003).

[13] T. Kimura, G. Lawes, T. Goto, Y. Tokura, and A. P. Ramirez, Phys. Rev. B 71, 224425 (2005).

[14] S. Heinze, K. von Bergmann, M. Menzel, J. Brede, A. Kubetzka, R. Wiesendanger, G. Bihlmayer, and S. Blügel, Nat. Phys. 7, 713 (2011).

[15] J. Sampaio, V. Cros, S. Rohart, A. Thiaville, and A. Fert, Nat. Nanotechnol. 8, 839 (2013).

[16] W. Jiang, P. Upadhyaya, W. Zhang, G. Yu, M. B. Jungfleisch, F. Y. Fradin, J. E. Pearson, Y. Tserkovnyak, K. L. Wang, O. Heinonen et al., Science 349, 283 (2015).
[17] Y. Yamasaki, S. Miyasaka, Y. Kaneko, J.-P. He, T. Arima, and Y. Tokura, Phys. Rev. Lett. 96, 207204 (2006).

[18] S. Ishiwata, Y. Taguchi, H. Murakawa, Y. Onose, and Y. Tokura, Science 319, 1643 (2008).

[19] H. Murakawa, Y. Onose, K. Ohgushi, S. Ishiwata, and Y. Tokura, J. Phys. Soc. Jpn. 77, 043709 (2008).

[20] A. V. Chubukov, D. V. Efremov, and I. Eremin, Phys. Rev. B 78, 134512 (2008).

[21] T. Frawley, R. Schoonmaker, S. H. Lee, C.-H. Du, P. Steadman, J. Strempfer, K. A. Ziq, S. J. Clark, T. Lancaster, and P. D. Hatton, Phys. Rev. B 95, 064424 (2017).

[22] X. Z. Yu, Y. Tokunaga, Y. Kaneko, W. Z. Zhang, K. Kimoto, Y. Matsui, Y. Taguchi, and Y. Tokura, Nat. Commun. 5, 3198 (2014).

[23] E. A. Giess, Science 208, 938 (1980).

[24] A. Hubert and R. Schäfer, Magnetic Domains: The Analysis of Magnetic Microstructures (Springer, Berlin, 2008).

[25] N. Nagaosa and Y. Tokura, Nat. Nanotechnol. 8, 899 (2013).

[26] D. N. Basov and A. V. Chubukov, Nat. Phys. 7, 272 (2011).

[27] M. Uchida, Y. Onose, Y. Matsui, and Y. Tokura, Science 311, 359 (2006).

[28] P. Milde, D. Köhler, J. Seidel, L. M. Eng, A. Bauer, A. Chacon, J. Kindervater, S. Mühlbauer, C. Pfleiderer, S. Buhrandt, C. Schütte, and A. Rosch, Science 340, 1076 (2013).

[29] O. Boulle, J. Vogel, H. Yang, S. Pizzini, D. de Souza Chaves, A. Locatelli, T. O. Mentes, A. Sala, L. D. Buda-Prejbeanu, O. Klein et al., Nat. Nanotechnol. 11, 449 (2016).

[30] S. Woo, K. Litzius, B. Krüger, M.-Y. Im, L. Caretta, K. Richter, M. Mann, A. Krone, R. M. Reeve, M. Weigand, P. Agrawal et al., Nat. Mater. 15, 501 (2016).

[31] B. T. M. Willis and C. J. Carlile, Experimental Neutron Scattering (Oxford University Press, Oxford, 2013).

[32] S. W. Lovesey and S. P. Collins, X-ray Scattering and Absorption by Magnetic Materials (Oxford University Press, Oxford, 2002). 
[33] J. Als-Nielsen and D. McMorrow, Elements of Modern X-ray Physics (Wiley, Chichester, 2010).

[34] J. Fink, E. Schierle, E. Weschke, and J. Geck, Rep. Prog. Phys. 76, 056502 (2013).

[35] H. Ohsumi and T.-H. Arima, Adv. Phys. X 1, 128 (2016).

[36] G. van der Laan and A. I. Figueroa, Coord. Chem. Rev. 277-278, 95 (2014).

[37] C. Sutter, G. Grübel, C. Vettier, F. de Bergevin, A. Stunault, D. Gibbs, and C. Giles, Phys. Rev. B 55, 954 (1997).

[38] H. A. Dürr, E. Dudzik, S. S. Dhesi, J. B. Goedkoop, G. van der Laan, M. Belakhovsky, C. Mocuta, A. Marty, and Y. Samson, Science 284, 2166 (1999).

[39] G. van der Laan, C. R. Phys. 9, 570 (2008).

[40] F. Fabrizi, H. C. Walker, L. Paolasini, F. de Bergevin, A. T. Boothroyd, D. Prabhakaran, and D. F. McMorrow, Phys. Rev. Lett. 102, 237205 (2009).

[41] A. M. Mulders, S. M. Lawrence, A. J. Princep, U. Staub, Y. Bodenthin, M. García-Fernández, M. Garganourakis, J. Hester, R. Macquart, and C. D. Ling, Phys. Rev. B 81, 092405 (2010).

[42] J. Als-Nielsen and D. McMorrow, Elements of Modern X-Ray Physics (Wiley, Chichester, 2001).

[43] T. Adams, A. Chacon, M. Wagner, A. Bauer, G. Brandl, B. Pedersen, H. Berger, P. Lemmens, and C. Pfleiderer, Phys. Rev. Lett. 108, 237204 (2012).
[44] S. L. Zhang, A. Bauer, H. Berger, C. Pfleiderer, G. van der Laan, and T. Hesjedal, Phys. Rev. B 93, 214420 (2016).

[45] J.-W. G. Bos, C. V. Colin, and T. T. M. Palstra, Phys. Rev. B 78, 094416 (2008).

[46] S. L. Zhang, G. van der Laan, and T. Hesjedal, Nat. Commun. 8, 14619 (2017).

[47] M. C. Langner, S. Roy, S. K. Mishra, J. C. T. Lee, X. W. Shi, M. A. Hossain, Y. D. Chuang, S. Seki, Y. Tokura, S. D. Kevan, and R. W. Schoenlein, Phys. Rev. Lett. 112, 167202 (2014).

[48] A. Frano, S. Blanco-Canosa, E. Schierle, Y. Lu, M. Wu, M. Bluschke, M. Minola, G. Christiani, H. U. Habermeier, G. Logvenov et al., Nat. Mater. 15, 831 (2016).

[49] A. K. Yadav, C. T. Nelson, S. L. Hsu, Z. Hong, J. D. Clarkson, C. M. Schlepetz, A. R. Damodaran, P. Shafer, E. Arenholz, L. R. Dedon et al., Nature (London) 530, 198 (2016).

[50] S. L. Zhang, I. Stasinopoulos, T. Lancaster, F. Xiao, A. Bauer, F. Rucker, A. A. Baker, A. I. Figueroa, Z. Salman, F. L. Pratt et al., Sci. Rep. 7, 123 (2017).

[51] J. P. Hannon, G. T. Trammell, M. Blume, and D. Gibbs, Phys. Rev. Lett. 61, 1245 (1988).

[52] M. Blume and D. Gibbs, Phys. Rev. B 37, 1779 (1988).

[53] J. P. Hill and D. F. McMorrow, Acta Crystallogr., Sect. A 52, 236 (1996). 